\title{
Der Löß in Bulgarien
}

\section{Von Em. Fotakiewa und M. Minkov (Sofia)}

\author{
Mit 3 Abbildungen und 1 Tabelle
}

$\mathrm{Zusammenf}$ assung. Der Löß in Bulgarien nimmt eine Fläche von $9800 \mathrm{~km}^{2}$ ein und ist ausschließlich in den Donaurandgebieten verbreitet. Er überdeckt mantelartig das ältere Relief - die pliozänen Denudations- und Sedimentationsoberflächen und pleistozänen Flußterrassen. Auf den Hochebenen liegt er auf Kreide-, Tertiär- und altpleistozänen Ablagerungen, von denen er durch eine aus rotbraunem, terrarossa-ähnlichem Lehm bestehende Verwitterungskruste getrennt ist. Auf den Terrassen liegt er auf altersunterschiedlichen Alluvialsedimenten.

Die Mächtigkeit der Lößformation hängt von der Entfernung von der Donau und dem Alter des geomorphologischen Elementes, dem es aufliegt, ab. Auf den in unmittelbarer Nähe der Donau liegenden Hochebenen beträgt diese Mächtigkeit zwischen 50 und $60 \mathrm{~m}$ (in Lößrücken bis zu $102 \mathrm{~m}$ ). Ungefähr $10 \mathrm{~km}$ südlich des Donauufers fällt diese Mächtigkeit schroff auf $25-30 \mathrm{~m}$ ab und erreicht in den südlichen Randgebieten kaum 4-5 m.

Mit der Entfernung von der Donau verändert sich auch die lithofaziale Zusammensetzung, wobei die einzelnen lithofazialen Varietäten territorial als schmale, unter sich und zur Donau parallel verlaufende Streifen in folgender Reihenfolge auftreten: lößartiger Sand, sandiger Löß, typischer Löß, lehmiger Löß und lößartiger Lehm.

Als Grundlage der Lößstratigraphie dienen die Fossilböden, die in bezug auf Zahl, Typ und räumliche Verbreitung hohe Beständigkeit aufweisen. Die Zahl der Lößhorizonte hängt vom Alter des Reliefelements, auf das sich der Lößkomplex ablagert, wie auch von den Erhaltungsbedingungen der primären Akkumulation ab. Auf den vorquartären und unterpleistozänen Reliefformen besteht der Lößkomplex aus sechs Lößhorizonten, die von fünf Fossilböden getrennt sind. Auf der 20-35-m-Terrasse (Riß/Würm I) finden sich drei Lößhorizonte, die durch zwei Fossilböden getrennt sind, auf der 12-15-m-Terrasse (Würm II) ein Lößhorizont.

Auf Grund der paläopedologischen, paläontologischen und vorgeschichtlichen Daten und des Prinzips der stratigraphischen Analogie nehmen wir an, daß der erste Lößhorizont während des Mindel I, der zweite im Mindel II, der dritte zur Rißeiszeit (I u. II) der vierte im Würm I, der fünfte im Würm II, der sechste im Würm III abgelagert worden sind, wobei der zweite und der dritte der Fossilböden interglaziale und die übrigen interstadiale Böden darstellen.

Die bisher durchgeführten Untersuchungen begründen die Annahme, daß der Löß in Bulgarien äolischen Ursprungs ist und aus den Überschwemmungsgebieten der Donau ausgeweht wurde.

$\mathrm{S} \mathrm{u} \mathrm{m}$ m a ry . The loess in Bulgaria takes up an area of $9,800 \mathrm{~km}^{2}$ and is distributed exclusively in the section along the Danube.

The loess forms a mantle over the pre-loessian relief surface forms - Pliocene denudation and sedimentation surfaces and Pleistocene river terraces. Loess formations overlie the Cretaceous, Tertiary and old Pleistocene deposits on the plateaux from which they are separated by a redbrown terra rossa-like clay weathering crust. On the terraces the loess overlies alluvial sediments of various ages, greater than its own age.

The thickness of the loess formation depends on the distance from the Danube river and the age of the geomorphologic element which it overlies. On the plateaux in immediate proximity to the Danube river this thickness varies within the range of 50 and $60 \mathrm{~m}$, loess walls reaching $102 \mathrm{~m}$. The thickness drops to $25-30 \mathrm{~m}$ about $10 \mathrm{~km}$ south of the Danube, whereas in the southern peripheral parts it hardly reaches $4-5 \mathrm{~m}$.

The lithofacial composition of the loess formation changes with recedance from the Danube, the individual faciel differences being territorially manifested as narrow strips parallel to each other and to the Danube in the following order: loessial sand, sandy loess, typical loess, clayey loess and loessial clay.

Loess stratigraphy is based on fossil soils, showing great stability with respect to number, type and territorial distribution. The number of loess horizons depends on the age of the relief element overlain by the loess complex and on the conditions favouring the preservation of the primary accumulation.

The loess complex, overlying pre-Quaternary and Lower-Pleistocene forms of the relief, consists of 6 loess horizons, separated by 5 fossil soils; on the terrace of a relative height of $25-35 \mathrm{~m}$ (Riss/Würm I) it consists of three loess horizons, separated by two fossil soils and on the terrace of a relative height of $12-15 \mathrm{~m}$ (Würm II) - by one loess horizon. 
On the basis of the available paleopedologic, paleontologic and pre-historic data and the principle of stratigraphic analogy we assume that the first loess horizon is deposited during Mindel I, the second-during Mindel II, the third-during Riss (I and II), the fourth-during Würm I, the fitth-during Würm II, the sixth-during Würm IIII, the second and third fossil soils being interglacial and the rest-interstadial.

The studies conducted so far provide grounds to assume that the loess in Bulgaria is of eolian origin and terrigenous source - the overflooding of the Danube river.

\section{Verbreitung}

Der Löß und die lößartigen Sedimente in Bulgarien nehmen eine Fläche von $9800 \mathrm{~km}^{2}$ ein, was rund $1 / 11$ vom Landesterritorium ausmacht (10). Sie sind ausschließlich in Nordbulgarien, und zwar in seinen Donau-Randgebieten, verbreitet (Abb. 1).
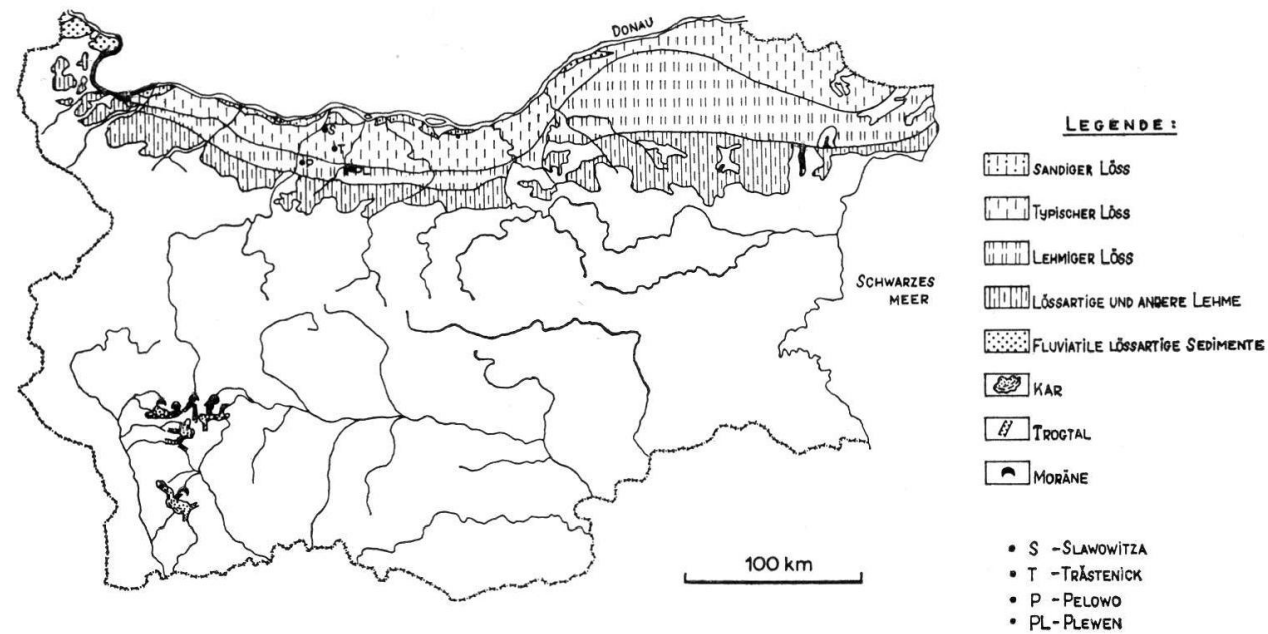

Abb. 1. Karte des Lösses und des Glazialreliefs in Bulgarien. Nach M. Minkov, K. StorLov und E. FotAKIEWA.

\section{Orographie, geologische Strukturund Geomorphologie des vom Löß eingenommenen Gebietes}

Die vom Löß eingenommene Fläche liegt gänzlich im morphotektonischen Gebiet, das unter dem Namen hügelige Donauebene bekannt ist. Letztere erstreckt sich nördlich des Stara-Planina-Vorgebirges, wo sich die letzten nördlichen Faltungen des Balkanidsystems verlieren. Sie besteht aus flachgelagerten und leichtgewellten mesozoischen, östlich vom Paläogen und Miozän und westlich vom Pliozän überdeckten Schichten. Die morphostrukturelle Entwicklung dieses Gebietes im Neogän war in den einzelnen Teilen nicht gleich. Westlich des Ogostaflusses hat sich im Pliozän das Becken vom Lom gebildet, das den südwestlichen Teil der Niederdonau-Senke darstellt. Am Anfang des Neogäns entstand östlich des Jantraflusses die flache, von den Anhöhen von Popowo, Razgrad und Samuilowo umrissene Wölbung von Razgrad. Ihre Aufwölbung hat den starken Einschnitt der Flüsse bedingt, welche tiefe cañon-ähnliche Täler und vereinzelte Hochebenen bilden. Die morphologische Entwicklung der einzelnen Teile der Ebene weist auch im Quartär gewisse Unterschiede auf. Im westlichen Teil (westlich des Iskarflusses) dominieren während des Unterquartärs die Akkumulationsprozesse. Von dem sich zu Beginn des Quartärs erhebenden Stara-Planina-Gebirge wurde hier unter den bedeutend feuchteren Klimaverhältnissen schotterlehmiges Material als einheitliche Uberdeckung abgelagert. Erst zur Zeit des Mittelpleistozäns schließt sich dieses Gebiet in die gesamte epirogene Erhebung des ganzen Niederdonau-Gebietes ein, und es bilden sich 5 Flußterrassen. 
Charakteristisch für die hiesigen Flußtäler ist ihr asymmetrisches Querprofil - sanfte linke und steile rechte Hänge. Der Zentralteil der Ebene (zwischen den Flüssen Iskăr und Jantra) wurde noch am Anfang des Quartärs von positiven Epirogenbewegungen erfaßt, und es bildeten sich 7 Flußterrassen. Das hydrographische Netz ist hier gut entwickelt. Die Täler sind breit und besitzen gut entwickelte Flußterrassen. Der asymmetrische Charakter nimmt in diesem Teil der Ebene allmählich ab und ist östlich des Witflusses nicht mehr ein kennzeichnendes Element ihrer Morphologie. Im östlichen Teil (östlich des Jantraflußtales) war die epirogene Hebung am stärksten, und die sich in die Kreidekalksteine einschneidenden Flüsse (Russenski Lom, Sucha Reka und Provadiiska - im oberen Verlauf) bilden cañon-ähnliche Täler. Im östlichsten Teil des Ludogorie nimmt die Hebung der hügeligen Donauebene $a b$ und geht allmählich in eine Senkung zum Küstenteil der Dobrudsha über $(2,7)$.

Orographisch wird die Donauebene durch ihr hügelig-ebenes Relief gekennzeichnet. Die mittlere Höhe über dem Meeresspiegel beträgt $176 \mathrm{~m}$, während sich die maximale absolute Höhe auf $502 \mathrm{~m}$ beläuft. Von der Gesamtfläche der Ebene zählen 61\% zum Höhengürtel von 0 bis $200 \mathrm{~m}$ und $39 \%$ zu demjenigen von 200 bis $500 \mathrm{~m}$ (7).

Das Klima ist kontinental, mit einer durchschnittlichen Jahrestemperatur von $11^{\circ}$, bei durchschnittlicher maximaler Monatstemperatur im Monat Juli von $23^{\circ}$ und minimaler im Monat Januar von $2^{\circ}$ und jährlichen Niederschlagsmengen von 500 bis $600 \mathrm{~mm}(7)$.

\section{Unterlage}

Der Löß überdeckt mantelartig die Erdoberflächenformen aus dem Vorlößrelief: sarmatisch-pontische Denudationsoberfläche, pliozäne Sedimentationsoberfläche, pliozäne Denudationsoberflächen, sowie pleistozäne Flußterrassen.

Die Meereshöhe, in der der Löß auftritt, schwankt vorwiegend von $0 \mathrm{~m}$ (Meeresspiegel bei Schabla) bis $245 \mathrm{~m}$ (die maximale Höhe der Lößrücken bei Swistov), wobei im Ludogorie stellenweise Lößflecken in einer Höhe von $350 \mathrm{~m}$ vorkommen.

Die Unterlage der Lößformation besteht aus Ablagerungen der Unteren Kreide (Barrême und Apt), der Oberen Kreide, Sarmat, Pliozän und Altpleistozän.

Auf den Hochebenen liegt die Lößformation unmittelbar auf alten, geringmächtigen Quartärschottern oder auf vorquartären Sedimenten. In beiden Fällen ist jedoch die Lößformation von der Unterlage durch eine aus rotbraunem, terrarossa-ähnlichem Lehm bestehende Verwitterungskruste getrennt, die infolge ihrer stratigraphischen Lage dem Pleistozän zugeordnet werden kann. Die Lößformation auf den Terrassen überdeckt die älteren altersverschiedenen Alluvialsedimente.

\section{Mächtigkeit}

Die Mächtigkeit der Lößformation hängt von der Entfernung von der Donau und dem Alter des geomorphologischen Elements, dem es aufliegt, ab. Die Entfaltung der primären Akkumulation ist nur auf den weiträumigeren Teilen der Sedimentations- oder Denudationsoberflächen sowie auf den breiten Terrassenniveaus erhalten geblieben, wo die Denudations- und vor allem die Erosionsprozesse während der Unterbrechungen der Lößanwehungen und nachher (in der Zeit des Holozäns) am schwächsten waren.

Die Mächtigkeit der Lößformation, unabhängig von der Erdoberflächenform, der sie aufliegt, ist entlang der Donau am größten und nimmt nach Süden zu allmählich ab. Am kennzeichnendsten in dieser Hinsicht sind die zwischen den nordbulgarischen Nebenflüssen der Donau eingeschlossenen Hochebenen. Überall auf dem nordwestlichen Winkel dieser Hochflächen sind hohe Lößrücken ausgebildet, die parallel zur Donau liegen, aber manchmal auch südlich-gerichtete, dem Nebenfluß parallel verlaufende, untergeordnete Ausläufer besitzen, wobei sie eine [-ähnliche Form erhalten. In den bestausgeprägten 
Lößrücken bewegt sich die Lößmächtigkeit zwischen 60 und $102 \mathrm{~m}$. Nur 8 bis $10 \mathrm{~km}$ südlich vom Donauufer fällt sie schroff auf $25-30 \mathrm{~m}$ ab. In den südlichen Randgebieten beträgt die Mächtigkeit kaum 4-5 m. Diese Gesetzmäßigkeit gilt nicht nur für den Komplex als Ganzes, sondern auch für die einzelnen Lößhorizonte, vor allem für die zwei jüngst abgelagerten, die die größte Mächtigkeit und die breiteste flächenmäßige Verbreitung aufweisen. Mit dem Näherkommen an die Donau nimmt die Mächtigkeit des Lösses auf den Flußterrassen die nordbulgarischen Flüsse entlang ebenfalls zu. Darauf ist die Zunahme ihrer relativen Höhe in nördlicher Richtung hin zurückzuführen (4, 2).

\section{Stratifikation und Stratigraphie}

G. Guntschew - Autor des ersten umfangreichen Werkes über den Löß in Bulgarien (4) - verzeichnet das Vorhandensein dreier altersverschiedener Lößhorizonte, die durch zwei Fossilböden voneinander getrennt sind. Er bezeichnet sie als Jung-, Mittel- und Altlöß und ist der Ansicht, daß der Junglöß in der zweiten Phase, der Mittellöß in der ersten Phase der Würm-Eiszeit, während der Altlöß in der Riß-Eiszeit abgelagert worden ist. Er bezweifelt das Vorhandensein eines vierten Lösses. D. JaRanov (8, 9, 10) bestimmt den Löß in Bulgarien als zur Würm-Eiszeit gehörend. Er nimmt an, daß in Bulgarien zwei Lösse vorliegen, von denen der ältere in der Würm-Eiszeit I und der jüngere in der Würm-Eiszeit II abgelagert worden ist; darunter verzeichnet er nur das Vorhandensein roter Grundlehme, die er als ein Ergebnis der Verwitterung geringmächtiger Lößüberdeckungen aus der Quartär-Anfangszeit hält. Auf Grund seiner Untersuchungen im Jahre 1960 stellte M. Minkov (12) in der Gegend von Lom das Vorhandensein von 5 Lößhorizonten fest, wobei er gleichzeitig verzeichnet, daß in der Gegend von Russe und Silistra 6 Lößhorizonte vorhanden sind. Im Zusammenhang mit dem in den letzten Jahren entfalteten Ausbau des Meliorationswesens wurden systematisch eingehende und zielgerichtete Untersuchungen des Lößkomplexes angestellt, die reichliches Licht auf die Stratifikation der Lößformation in Bulgarien geworfen haben.

Als Grundlage der Lößstratigraphie in Nordbulgarien dienen die Fossilböden, die in Bezug auf ihre Zahl, ihren Typ und ihre räumliche Verbreitung hohe Beständigkeit aufweisen. Die Zahl der Lößhorizonte hängt vom Alter des Reliefelements (Abb. 2) ab, auf das sich der Lößkomplex abgelagert hat, wie auch von den das Erhalten der primären Akkumulation begünstigenden Bedingungen. Auf den besterhaltenen Teilen der älteren Reliefformen (vorquartäre und unterpleistozäne) wird ein volles Profil des Lößkomplexes beobachtet. Auf diesen Reliefformen stellt man 6 Lößhorizonte $\left(\mathrm{L}_{1} \ldots \mathrm{L}_{6}\right)$ fest, von denen ein jeder oberflächlich bodentragend $\left(\mathrm{FB}_{1} \ldots \mathrm{FB}_{6}\right)$ ist. Nahe der Donau ist der dritte Fossilboden $\mathrm{FB}_{3}$ geteilt, d. h. unmittelbar übereinander sind zwei selbständige Profile $\left(\mathrm{FB}_{3} \mathrm{a}\right.$ und $\mathrm{FB}_{3} \mathrm{~b}$ ) ausgebildet, was für eine vorübergehende Lößanwehung in diesem Gebiet zur Zeit der dritten Fossilbodenformierung $\left(\mathrm{FB}_{3}\right)$ spricht. Eine ähnliche Teilung wird stellenweise auch im fünften Fossilboden $\left(\mathrm{FB}_{5}\right)$ beobachtet.

Auf der Terrasse mit relativer Höhe von $22-35 \mathrm{~m}$, die sich, wie man annimmt, vor Antritt der Würm-Eiszeit gebildet hat, sind drei (durch zwei Fossilböden getrennte) Lößhorizonte abgelagert $\left(\mathrm{L}_{4}, \mathrm{~L}_{5}\right.$ und $\mathrm{L}_{6}$ ), während auf der Terrasse mit einer Relativhöhe von $12-15 \mathrm{~m}$, die wahrscheinlich vom Würm II datiert, sich ein Lößhorizont angelagert hat, auf dem der heutige Boden ausgebildet ist. Die mächtigsten Lößhorizonte sind der dritte $\left(\mathrm{L}_{3}\right)$, fünfte $\left(\mathrm{L}_{5}\right)$ und sechste $\left(\mathrm{L}_{6}\right)$, während die übrigen $\left(\mathrm{L}_{1}, \mathrm{~L}_{2} \mathrm{u}\right.$. $\left.\mathrm{L}_{4}\right)$ von geringerer Mächtigkeit und durch die bodenbildenden Prozesse stark verändert sind. Den zweiten $\left(\mathrm{FB}_{2}\right)$, dritten $\left(\mathrm{FB}_{3} \mathrm{a}\right)$ und vierten $\left(\mathrm{FB}_{4}\right)$ Fossilboden stellen Waldböden dar, von denen der zweite $\left(\mathrm{FB}_{2}\right)$ die größte Differenziertheit in der mechanischen Zusammensetzung aufweist, während die übrigen Tschernoseme sind.

Obgleich die Schichtenfolge der Löß-Serien in Bulgarien gut geklärt ist, steht die Frage nach der stratigraphischen Eingliederung noch offen. Paläontologische und vorge- 


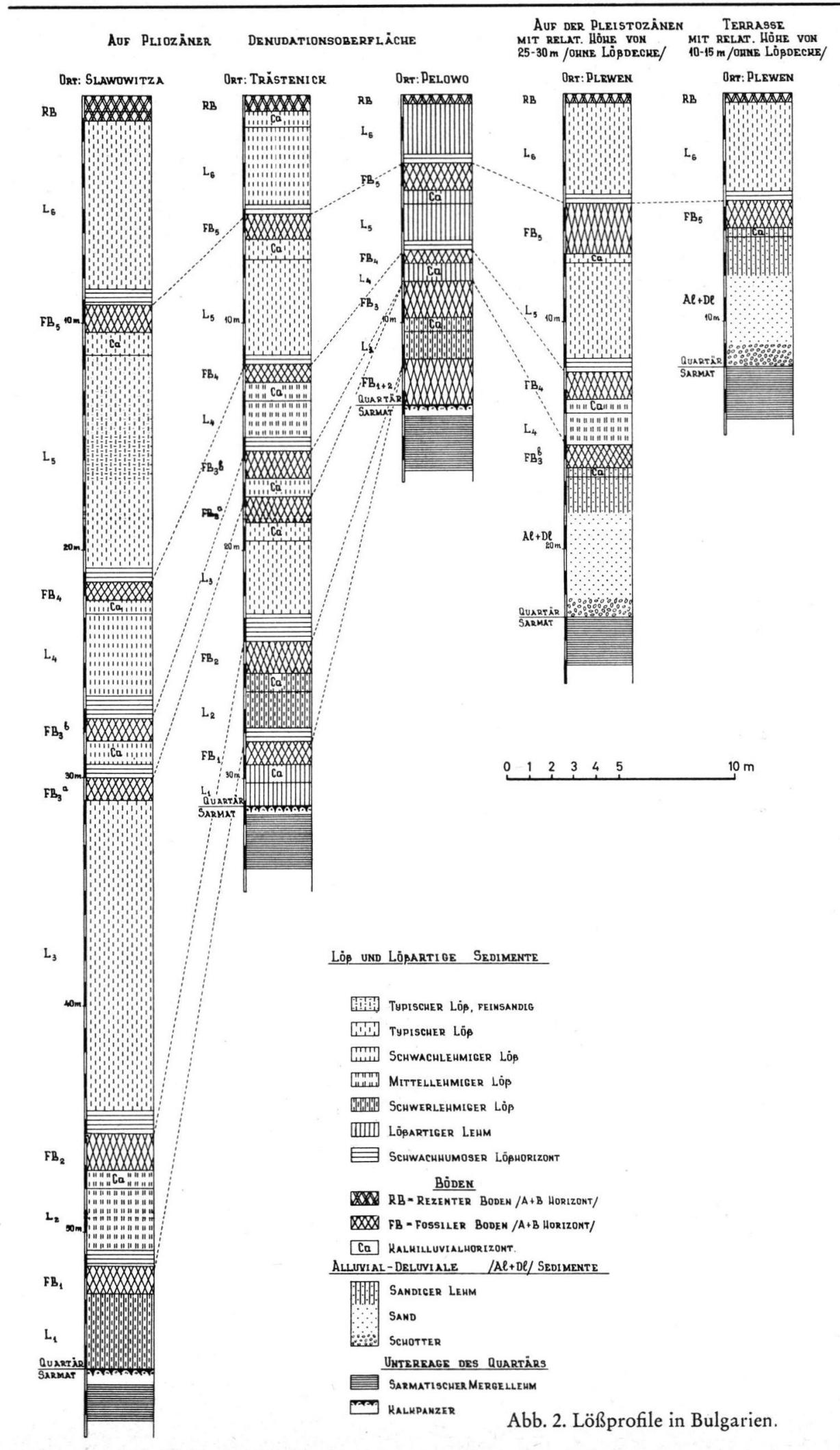


schichtliche Daten, wie die Funde an Elephas primigenius Buum. und Rhinoceros tichorbinus, wie auch Funde an vorgeschichtlichen steinernen Werkzeugen, berechtigen, das Alter der oberen zwei Lößhorizonte $\left(\mathrm{L}_{5}\right.$ und $\left.\mathrm{L}_{6}\right)$ als zum Würm gehörend zu bestimmen. Zur Datierung der übrigen Horizonte verfügen wir jedoch zur Zeit über keine Anhaltspunkte. In der xerothermen Gastropodenfauna, an der der Löß überreich ist, liegen keine Leitfossilien vor, weshalb sie zu diesem $Z$ wecke nicht benützt werden kann.

Auf Grund der vorliegenden Daten (paläopedologische, paläontologische und vorgeschichtliche) und des Prinzips der stratigraphischen Analogie wird vorerst angenommen, daß der erste Lößhorizont $\left(L_{1}\right)$ während des Mindel I, der zweite $\left(L_{2}\right)$ im Mindel II, der dritte $\left(\mathrm{L}_{3}\right)$ zur Riß-Eiszeit (I+II), der vierte $\left(\mathrm{L}_{4}\right)$ im Würm I, der fünfte $\left(\mathrm{L}_{5}\right)$ im Würm II, der sechste $\left(\mathrm{L}_{6}\right)$ im Würm III abgelagert worden sind, wobei der zweite $\left(\mathrm{FB}_{2}\right)$ und der dritte $\left(\mathrm{FB}_{3} \mathrm{a}\right)$ der Fossilböden interglaziale und die übrigen interstadiale Böden darstellen.

Korngrößenzusammensetzungund fazielle Veränderungen

Kennzeichnend für die Lößzusammensetzung und Bestimmung der Gesetzmäßigkeiten in seinen räumlichen Veränderungen ist das Verhältnis zwischen den wichtigsten drei Fraktionen und zwar der Sand - $\left(D_{1} ; 0,1-0,05 \mathrm{~mm}\right)$, Staub - $\left(D_{2} ; 0,05-0,005 \mathrm{~mm}\right)$ und Tonfraktion $\left(D_{3} ;\langle 0,005 \mathrm{~mm})\right.$. Die granulometrische Zusammensetzung wurde nach dem aräometrischen Verfahren durch mechanische Bearbeitung und Sieden der Proben mit 25\% \%iger Ammoniaklösung im Laufe einer Stunde bestimmt.

Diese Verhältnisse sind im auf Grund analytischer Daten aus hunderten Proben zusammengestellten Dreieckdiagramm (Abb. 3) wiedergegeben. Aus demselben können zwei wichtige Schlußfolgerungen gezogen werden. 1) Die subaquatischen (alluvialen und deluvialen) lößartigen Sedimente (Gruppe VI auf Abb. 3) besitzen eigene charakteristische, sich völlig von den subaerischen unterscheidende granulometrische Linien. 2) Letztere

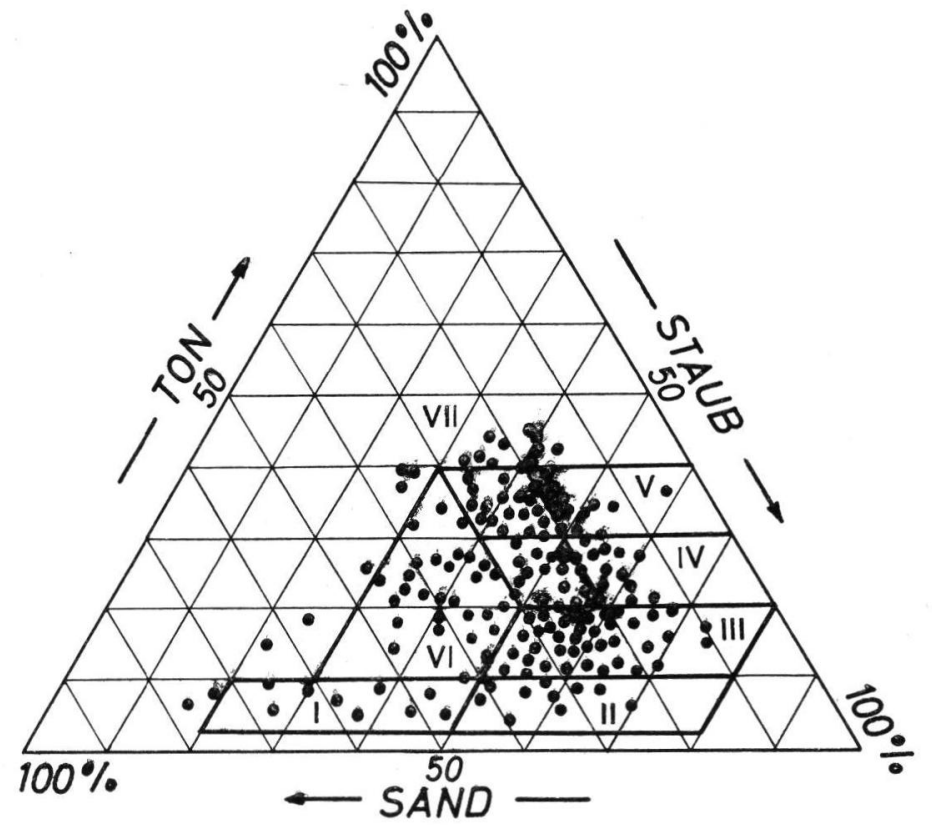

Abb. 3. Granulometrische Zusammensetzung bulgarischer Lösse. $I=$ lößartiger Sand, II = sandiger Löß, III = typischer Löß, IV = alluviale und deluviale lößähnliche Sedimente, VII = sandige und andere Lehme. 
zeigen eine allmähliche Veränderung in der Zusammensetzung von Sand- über Staub- zu Tonarten, was vom Vorhandensein fazieller Veränderungen in der Lößformation spricht. Letztere kommen zur Zeit in Bulgarien durch die Klassifikation der Tab. 1 zum Ausdruck.

Tabelle 1

\begin{tabular}{|c|c|c|c|c|c|c|}
\hline Gruppe & \multicolumn{6}{|c|}{ Lößgesteine } \\
\hline Untergruppe & \multicolumn{3}{|c|}{ Lößartige } & \multicolumn{3}{|c|}{ I.̈ßß } \\
\hline Genesis & \multirow{2}{*}{$\begin{array}{c}\text { alluvial- } \\
\text { deluviale } \\
\text { u. a. }\end{array}$} & \multicolumn{5}{|c|}{ äolische lößartige Gesteine } \\
\hline Varietät & & $\begin{array}{c}\text { lößartiger } \\
\text { Lehm }\end{array}$ & $\begin{array}{c}\text { lößartiger } \\
\text { Sand }\end{array}$ & $\begin{array}{c}\text { sandiger } \\
\text { Löß }\end{array}$ & $\begin{array}{c}\text { typischer } \\
\text { Löß }\end{array}$ & $\begin{array}{c}\text { lehmiger } \\
\text { Löß }\end{array}$ \\
\hline $\begin{array}{l}\text { Grenzwerte d. } \\
\text { Fraktionen } \\
\text { in } \% \\
\quad \text { Sand } \\
0,25-0,05 \mathrm{~mm}\end{array}$ & $3 c-60$ & $<30$ & $40-77$ & $10-47$ & $<40$ & $<30$ \\
\hline $\begin{array}{c}\text { Staub } \\
0,05-0,005 \\
\mathrm{~mm}\end{array}$ & $30-50$ & $>30$ & $20-30$ & $50-80$ & $50-80$ & $>40$ \\
\hline $\begin{array}{c}\text { Ton } \\
<0,005 \mathrm{~mm}\end{array}$ & $10-40$ & $30-40$ & $3-10$ & $3-10$ & $10-20$ & $20-30$ \\
\hline
\end{tabular}

Die erste grundlegende Gesetzmäßigkeit in der Veränderung der granulometrischen Zusammensetzung der Lößformation in Bulgarien ist die allmähliche Zunahme der Tonmenge und die Veränderung des Verhältnisses zwischen den einzelnen Fraktionen mit zunehmender Entfernung von der Donau. Das bestimmt die für den Niederdonau-Löß charakteristische Verlehmung mit zunehmender Entfernung vom terrigenen Ursprung (Donau) und den allmählichen Übergang der Lößfraktion in lößartigen Lehm. Die Entfernungen, in denen diese Veränderungen auftreten, sind jedoch nicht überall gleich. Im westlichen Teil der Ebene ändert sich das Verhältnis zwischen den Fraktionen in schmalen Streifen, die sich in folgender Reihenfolge anordnen lassen. Am Ufer ist sandiger Löß abgelagert, der nach nur $2-3 \mathrm{~km}$ in Staublöß und nach $8-10 \mathrm{~km}$ in lehmigen Löß übergeht, um sich nach einer Entfernung von $16-20 \mathrm{~km}$ zu verjüngen und in lößartigen Lehm zu verwandeln. O'stlich - im Zentralteil der Ebene - sind im Zusammenhang mit der Verbreitung des Lößgebietes die Übergänge fließender und treten in größeren Entfernungen auf. Im Gebiet von Russe wird das Übergangsgebiet schmaler, die Übergänge behalten jedoch ihren allmählichen Charakter bei, der in den großen Lößflächen der Dobrudsha am besten zum Ausdruck kommt (14). ${ }^{1}$ )

Infolge dieser Gesetzmäßigkeit in der Veränderung der lithofaziellen Zusammensetzung der Lößformation in Richtung Nord-Süd ordnen sich die Varietäten territorial als schmale, unter sich und der Donau parallel verlaufende Streifen in folgender Reihenfolge an: lößartiger Sand, sandiger Löß, typischer Löß, lehmiger Löß und lößartiger Lehm.

1) Der Unterschied in der "Geschwindigkeit" der Veränderungen kommt durch den mathematischen Ausdruck der mengenmäßigen Zunahme der Tonfraktion $\left(\mathrm{D}_{3}\right)$ in Abhängigkeit von der Entfernung (1) von der Donau zutage. Im westlichen Teil, bis zum Iskarfluß, hat diese Abhängigkeit $\mathrm{D}_{3}=$ (1) logarithmischen Charakter der Art $\mathrm{y}=\mathrm{a} \lg \mathrm{x}+\mathrm{b}$, östlich hingegen linearen der Art $y=a x+b$. 
Die zweite wichtige Gesetzmäßigkeit in der Veränderung der granulometrischen Zusammensetzung des Lösses auf dem Territorium von Bulgarien ist die allmähliche Zunahme des tonigen und Abnahme des sandigen Inhalts in west-östlicher Richtung. Eine gewisse Abweichung davon wird im Gebiet von Silistra und in südöstlicher Richtung nach Kranewo und Telerig beobachtet, wo das Vorhandensein von Staublöß $\left(\mathrm{D}_{2}>60 \%\right)$ den Übergang in ein neues Akkumulationsgebiet mit anderer Transportrichtung kennzeichnet.

Gewissen Veränderungen in bezug auf ihre Zusammensetzung unterliegt die Lößformation auch nach der Tiefe zu. Diese Veränderungen stehen in engem Zusammenhang mit der Stratifikation des Lößkomplexes und sind komplizierten Charakters, da sie von Gründen verschiedener Natur und Zeitdauer verursacht worden sind.

Die erste Gesetzmäßigkeit kommt hier in der sinusoidalen Veränderung der durchschnittlichen Korngröße in den einzelnen Lößhorizonten zum Ausdruck, wobei die Menge der gröber granulierten Fraktionen nach der Horizontmitte zu am größten ist. Diese Zusammensetzungs- und gleichzeitig auch Struktursymmetrie ist für die Veränderungen in den Sedimentationsverhältnissen im Verlauf einer Lößablagerung kennzeichnend, wobei die Zunahme der durchschnittlichen Korngröße nach der Horizontmitte $\mathrm{zu}$ - bei völliger Abwesenheit phytogener Makroporen - für eine maximale Klimaaridität spricht, die für ein völliges Unterbrechen des Pflanzenwuchses in der Mitte der Glazialperiode kennzeichnend ist. Dabei ist der Inhalt der tonigen Fraktion in den Fossilböden am größten, bei denen die Menge dieser Fraktionen von 5 bis 15\% größer ist als diejenige des Lößhorizontes, auf der der Boden ausgebildet ist.

Die vergleichende Gegenüberstellung der granulometrischen Zusammensetzung des Lösses der einzelnen Lößbildungsstadien zeigt, daß die grobkörnigste (staub-sandige) Feinerde während des dritten $\left(\mathrm{L}_{3}\right)$ und fünften $\left(\mathrm{L}_{5}\right)$ lößbildenden Stadiums abgelagert worden ist. Die übrigen Lößhorizonte $\left(\mathrm{L}_{1}, \mathrm{~L}_{2}, \mathrm{~L}_{4}\right.$ und $\left.\mathrm{L}_{6}\right)$ sind bei geringer Transportkraft und größerem Materialdefizit im Deflationsgebiet abgelagert. Dabei ist der $\mathrm{L}_{5}$ immer staub-sandiger als der $\mathrm{L}_{6}$, besitzt ein weiteres Verbreitungsareal, und die Übergänge von einer Varietät zur anderen finden auf größeren Horizontalentfernungen statt.

\section{Ursprung des Lösses}

Der erste Forscher des Lösses in Bulgarien, G. Guntschev (4), vertritt noch im Jahre 1935 die Ansicht, daß der Löß in Bulgarien äolischen Ursprungs ist und nimmt an, daß der Lößstaub vom periglazialen Gebiet des nordeuropäischen Gletschers her zur Zeit der quartären Vereisungen angeweht worden ist. Diese Ansicht ist auch von P. BoJKov (1) und D. Jaranov $(8,9)$ übernommen worden. Einige Jahre später (1939) wurde vom Botaniker B. Stefanov $(16,17)$ die Meinung lanciert, daß der Löß in Süßgewässern abgelagert worden sei, und daß die Lößablagerung eine Fortsetzung des pliozänen Sedimentationszyklus darstelle. Dieser Auffassung schloß sich niemand an, und bis zum Jahre 1949 herrscht in den Kreisen der bulgarischen Spezialisten - J. Galabov (5, 6), K. KR $\breve{S T E V}$ (11) u. a. - völlige Einstimmigkeit über die Frage des Lößursprungs, nämlich daß er ein Ergebnis äolischer Tätigkeit ist. Im Jahre 1949 veröffentlichte I. P. Gerassimov die Studie "Geomorphologische Beobachtungen in Bulgarien“(3), wo er auf Grund seiner Wahrnehmungen, daß „der typische Löß in Nordbulgarien eine beschränkte Verbreitung und geringe Mächtigkeit (nicht mehr als 15-20 m) aufweist“ und daß ,in ihm streng stratigraphische, durch überdeckte Böden getrennte Horizonte fehlen “, die Ansicht vertritt, daß „der Löß und die lößartigen Ablagerungen in Nordbulgarien ein Ergebnis der neuesten, quartären Verwitterung von Ablagerungen unterschiedlichster Genesis - eluviale, alluviale u. a. - sind, die unter der Einwirkung der sialitisch-karbonatischen Verwitterung einen einheitlichen lithologischen Charakter erhalten haben". Eine in bezug auf den äolischen Ursprung des Lösses in Bulgarien entgegengesetzte Meinung vertritt K. Mischev (15). Er nimmt an, daß derselbe ein Ergebnis der Wassersedimentation ist. 
Auf Grund der vom geologischen Kollektiv beim „Energohydroprojekt" gesammelten Materialien im Jahre 1956, veröffentlichte D. JARANov (10) eine verallgemeinernde Studie über den Löß in Bulgarien, in welcher er wieder den Standpunkt über den äolischen Ursprung desselben vertritt. Als Ursprung des Lößstaubes sieht er die Donauüberschwemmungen an.

Die äolische Theorie über den Ursprung des bulgarischen Lösses wurde jedoch am besten von M. Minkov (12) begründet. Als wichtigste Beweise zugunsten dieser Theorie können gegenwärtig folgende genannt werden:

1. Die mantelartige Ablagerung der Lößformation auf altersunterschiedlichen Felsen und Formen.

2. Das Vorhandensein eines stark gegliederten, überdeckten hydrographischen Netzes.

3. Die große Mächtigkeit der Lößdecke in unmittelbarer Nähe der Donau und ihre beschleunigte Abnahme nach Süden zu.

4. Die südliche Neigung der Hochebenen und Lößterrassen in der Lößzone bei nördlicher Neigung der Unterlage.

5. Das Vorhandensein von Lößrücken, dünenähnlichen Formen und abflußlosen Niederungen im gegenwärtigen Relief.

6. Das Vorhandensein von Fossilböden im Löß.

7. Das Vorhandensein von querer, schräger und großwelliger Schichtung im sandigen Löß an der Donau.

8. Das Vorhandensein von zwischengeschalteten Dünen im Löß.

9. Die staubartige, sehr gleichartige Zusammensetzung, wobei die Körnigkeit nach Süden zu allmählich feiner wird.

10. Zunahme der Tonkomponente im Altlöß und den Basishorizonten (im Gegensatz zum Grundgesetz der Wassersedimentation).

11. Der Unterschied in der mineralogischen Zusammensetzung im Vergleich zur Unterlage. Fauna.

12. Das Vorhandensein xerothermer Gastropodenfauna; das Fehlen aquatischer

13. Die Funde von Festlandsäugetieren und archäologischen Artefakten.

Der äolische Ursprung unseres Lösses wird gegenwärtig von fast sämtlichen Forschern des Quartärs in Bulgarien angenommen.

\section{$\mathrm{Sch}$ if $\mathrm{t} \mathrm{tum}$}

1. Bojkov, P.: Der Löß von Nordbulgarien und die auf ihm gebildeten Böden. Ztschr. bulg. geol. Ges. 8, 1, 1936.

2. Fotakiewa, E.: Geomorphologische Bedingtheit der Bodenunterschiede im Mittelteil der Donauebene (zwischen den Flüssen Osăm und Jantra). Materialien VIII. Internat. Kongr. Bodenkd. 5, Bukarest 1964.

3. Gerassimov, I. P.: Geomorphologische Beobachtungen in Bulgarien. Probl. phys. Geogr. 14, Moskau, Akad. Wiss. UdSSR, 1949.

4. Guntschev, G.: Der Löß in Nordbulgarien. Mitt. bulg. geogr. Ges. 3, 1935.

5. GĂLĂвOV, J.: Kurze phys.-geographische Charakteristik Bulgariens. Jb. Direktion geol. u. Grubenforsch., A, 4, 1946.

6. GĂLĂвоv, J.: Quartärablagerungen und Quartärmorphologie. Jb. Direktion geol. u. Grubenforsch., A, 4, 1946.

7. Gălăbov, J., Iwanov, Il,, Pentschev, P., Mischev, K., Nedeltschewa, W.: Physik. Geographie Bulgariens, 1956. 
8. Jaranov, D.: Entwicklung des geomorphologischen Studiums und die wichtigsten geomorphologischen Probleme der Balkanhalbinsel. Jb. Sofioter Univ., Histor.-Philolog. Fakultät 33, Sofia 1937.

9. Jaranov, D.: Les zones morphologiques dans les parties centrales et orientales de la Peninsule Balkanique. C. R. IV-iéme Congr. géogr. et des ethnogr. slaves, Sofia 1936.

10. Jaranov, D.: Der Löß und die lößartigen Sedimente in Bulgarien. Mitt. Bod. Instituts, BAW, III, Sofia 1956.

11. Krăstev, K.: Das Lößrelief in Nordwestbulgarien. Mitt. Semin. b. d. Hist.-Philolog. Fakult. Sofioter Univ., I, 1942.

12. Minkov, M.: Der Löß und die lößartigen Sedimente zwischen den Flüssen Skomlja und Ogosta. Wiss. Arbeiten Geol. Bulgariens, Schriftenreihe Stratigraphie u. Tektonik, I, 1960.

13. Minkov, M.: Physiko-mechanische Eigenschaften der Lößgesteine zwischen den Flüssen Skomlja und Ogosta. Wiss. Arbeiten Geol. Bulgariens. Schriftenreihe Ingenieur-Geol. u. Hydro-Geol., II, 1963.

14. Mrnkov, M.: Der Löß Nordbulgariens (kompl. Studie). (Mskr.).

15. Mischev, K.: Geomorphologische Forschungen der hügeligen Donauebene zwischen den Flüssen Widbol und Ogosta. Mitt. Geogr. Inst., BAW 4, 1959.

16. Stefanov, B.: Der Löß und die Verbreitung der Walddecke in der Donauer Randebene. Mitt. bulg. geogr. Ges., VI, 1939.

17. Stefanov, B.: Zur Frage des Ursprungs und des Alters des Lösses in der Donauer Randebene. Jb. Sofioter Univ., Landw.-Forst. Fakultät, 18, 1939/40.

Manuskr. eingeg. 24. 7. 1965.

Anschriften der Verf.: Dr. E. Fotakiewa, N. Poushkarov-Institut, 136, 9 Sept. Boul., Sofia (Bulg.) Minko Minkov, Geol. Inst. d. Bulgar. Akademie d. Wissensch., Sofia (Bulgarien). 